\title{
Observation of Attractive and Repulsive Polarons in a Bose-Einstein Condensate
}

\author{
Nils B. Jørgensen, ${ }^{1}$ Lars Wacker, ${ }^{1}$ Kristoffer T. Skalmstang, ${ }^{1}$ Meera M. Parish, ${ }^{2}$ Jesper Levinsen, ${ }^{2}$ \\ Rasmus S. Christensen, ${ }^{1}$ Georg M. Bruun, ${ }^{1}$ and Jan J. Arlt ${ }^{1}$ \\ ${ }^{1}$ Institut for Fysik og Astronomi, Aarhus Universitet, 8000 Aarhus C, Denmark \\ ${ }^{2}$ School of Physics \& Astronomy, Monash University, Victoria 3800, Australia
}

(Received 17 May 2016; revised manuscript received 13 June 2016; published 28 July 2016)

\begin{abstract}
The problem of an impurity particle moving through a bosonic medium plays a fundamental role in physics. However, the canonical scenario of a mobile impurity immersed in a Bose-Einstein condensate (BEC) has not yet been realized. Here, we use radio frequency spectroscopy of ultracold bosonic ${ }^{39} \mathrm{~K}$ atoms to experimentally demonstrate the existence of a well-defined quasiparticle state of an impurity interacting with a BEC. We measure the energy of the impurity both for attractive and repulsive interactions, and find excellent agreement with theories that incorporate three-body correlations, both in the weak-coupling limits and across unitarity. The spectral response consists of a well-defined quasiparticle peak at weak coupling, while for increasing interaction strength, the spectrum is strongly broadened and becomes dominated by the many-body continuum of excited states. Crucially, no significant effects of three-body decay are observed. Our results open up exciting prospects for studying mobile impurities in a bosonic environment and strongly interacting Bose systems in general.
\end{abstract}

DOI: 10.1103/PhysRevLett.117.055302

The behavior of a mobile impurity interacting with its environment has provided deep insight into quantum manybody systems. Since Landau and Pekar first proposed that the coupling between electrons and lattice phonons leads to the existence of quasiparticles termed polarons [1], this idea has systematically been developed [2]. The concept of the polaron is now central to our understanding of a wide range of systems, including technologically important semiconductors [3], ${ }^{3} \mathrm{He}-{ }^{4} \mathrm{He}$ mixtures [4], and high temperature superconductors [5]. Indeed, even elementary particles of the standard model acquire their mass by coupling to the bosonic Higgs particle.

Because of the great flexibility of atomic gases, the experimental realization of the impurity problem in a degenerate Fermi gas [6-8] has led to a dramatic improvement in our theoretical understanding of this fundamental problem [9-16]. The bosonic counterpart has been subject of intense theoretical investigation [17-25] and some specific cases have been studied experimentally: impurities interacting with an uncondensed bosonic medium [26], charged or fixed impurities in a Bose-Einstein condensate (BEC) [27-31], and impurities confined to a lattice [32]. However, despite the importance of the impurity problem in a bosonic reservoir, there has not yet been a realization of the canonical mobile impurity in a BEC-the Bose polaron.

We investigate the Bose polaron using a harmonically trapped BEC of ${ }^{39} \mathrm{~K}$ atoms initially prepared in the $|1\rangle \equiv$ $\left|F=1, m_{F}=-1\right\rangle$ state. To introduce impurities, we apply a radio frequency (rf) pulse, which transfers a small fraction of atoms into the $|2\rangle \equiv|1,0\rangle$ state, such that they can be regarded as isolated mobile impurities. The transition frequency $\omega_{\mathrm{rf}}$ is changed from its unperturbed value $\omega_{0}$ due to the impurity-BEC interaction. This interaction, characterized by the $s$-wave scattering length $a$, is highly tuneable using a Feshbach resonance. Thus, spectroscopy of the impurity state is performed, as shown schematically in Fig. 1. We measure the energy of the impurity state and find a well-defined Bose polaron both for attractive and repulsive interactions, in good agreement with theories that incorporate three-body correlations. This constitutes the first observation of the Bose polaron for both attractive and repulsive interactions, in parallel with work at JILA [33].

Figure 1 illustrates the behavior of the zero-momentum impurity in a uniform BEC. We parametrize the interaction strength as $k_{n} a$ with the wave number $k_{n}=\left(6 \pi^{2} n_{0}\right)^{1 / 3}$, where $n_{0}$ is the average BEC density. The energy scale of the system is $E_{n}=\hbar^{2} k_{n}^{2} / 2 m$, where $m$ is the mass of ${ }^{39} \mathrm{~K}$. For weak interactions, $1 / k_{n} a \ll-1$ and $1 / k_{n} a \gg 1$, the impurity forms well-defined quasiparticle states termed attractive and repulsive polaron, respectively. These have mean-field energy $4 \pi \hbar^{2} n_{0} a / m$, plus medium corrections that have recently been determined up to order $a^{3}$ [24]. On the attractive side of the Feshbach resonance, the zero-momentum attractive polaron is the ground state. In the absence of Efimov physics [34-36], the attractive polaron state exhibits an avoided crossing with the molecular state beyond unitarity [20,25]. Above the ground state there is a continuum of many-body states, which in the weakly interacting limit is formed by polarons and Bogoliubov excitations with zero total momentum (see Supplemental Material [37]). On the repulsive side $1 / k_{n} a>0$, the polaron becomes increasingly damped when approaching the Feshbach resonance, since it can decay into a continuum of lower lying manybody states, and it is inherently metastable.

The experimental apparatus used to produce BECs is described in detail in [38]. Briefly, a dual-species 


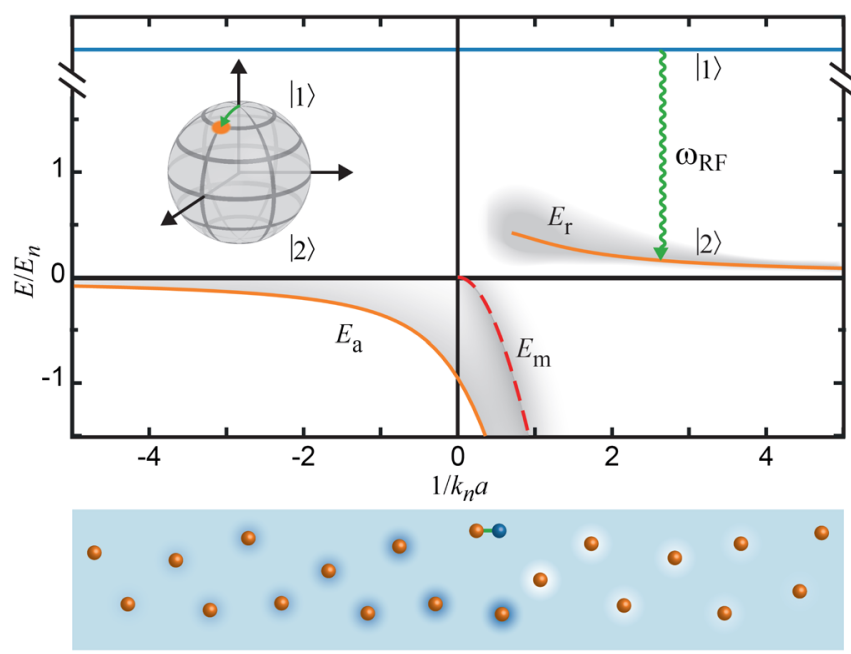

FIG. 1. Sketch of the spectroscopic method and the impurity energy spectrum. A radio frequency pulse transfers atoms from the $|1\rangle$ to the $|2\rangle$ state. Only a small fraction is transferred, corresponding to a rotation by a small angle on the Bloch sphere (inset) in a noninteracting system. The solid lines show the energies of the zero-momentum attractive $\left(E_{a}\right)$ and repulsive $\left(E_{r}\right)$ polaron states in a uniform BEC as a function of the interaction parameter $1 / k_{n} a$ (see text). The dashed line shows the molecular binding energy $E_{m}$ on the repulsive side of the Feshbach resonance, and the gray shading denotes a continuum of many-body states. The bottom cartoon shows impurity atoms (orange) in a BEC (blue); the intensity of the background color indicates the change in the BEC density due to the presence of impurity atoms.

magneto-optical trap captures ${ }^{87} \mathrm{Rb}$ and ${ }^{39} \mathrm{~K}$ atoms and subsequently evaporative cooling is performed in a magnetic trap. All ${ }^{87} \mathrm{Rb}$ atoms are evaporated leading to sympathetic cooling of ${ }^{39} \mathrm{~K}$. The remaining ${ }^{39} \mathrm{~K}$ atoms are loaded into an optical dipole trap consisting of two crossed beams at a wavelength of $1064 \mathrm{~nm}$. Two rapid adiabatic passages prepare the atoms in the $|1\rangle$ state by the transfers $|2,2\rangle \rightarrow$ $|2,-2\rangle$ and $|2,-2\rangle \rightarrow|1,-1\rangle$. This path ensures that no atoms are produced in the $|2\rangle$ state due to a potentially imperfect state preparation. The sample is then further evaporatively cooled by lowering the dipole trap power. During this evaporation, a Feshbach resonance at $33.6 \mathrm{G}$ is addressed to ensure efficient rethermalization. When a sufficiently low temperature is reached, the magnetic field is ramped to the desired value in the vicinity of the interstate Feshbach resonance located at $113.8 \mathrm{G}[37,39]$. Finally, the power of the dipole trap is raised to increase the density of the BEC, which results in trap frequencies of $\nu_{x}=158 \mathrm{~Hz}$, $\nu_{y}=167 \mathrm{~Hz}$, and $\nu_{z}=228 \mathrm{~Hz}$. At this point, the BEC consists of $2 \times 10^{4}$ atoms with average density $n_{0}=2.3 \times$ $10^{14} \mathrm{~cm}^{-3}$ at a temperature $T=160 \mathrm{nK}$ corresponding to $T / T_{c} \approx 0.6$ where $T_{c}$ is the critical temperature of BoseEinstein condensation. The $|1\rangle$ atoms are weakly interacting with scattering length $a_{B} \approx 9 a_{0}$, where $a_{0}$ is the Bohr radius, such that $k_{n} a_{B} \approx 0.01$.

To form the polaron, a square if pulse of $100 \mu$ s duration is used, which transfers a small fraction of atoms into the
12) state. This scheme of direct transfer ensures a perfect spatial overlap of impurities with the BEC. Furthermore, it is unique to a bosonic system since interaction effects in a Fermi gas subjected to a rf pulse are suppressed due to the Pauli principle [40]. The pulse length and experimental magnetic field precision result in a spectral full width at half maximum of $0.15 E_{n}$.

The rf transfer is described by the operator $H_{\mathrm{rf}}=$ $\Omega e^{-i \omega_{\mathrm{ri}} t} \sum_{\mathbf{k}} a_{\mathbf{k} 2}^{\dagger} a_{\mathbf{k} 1}+$ H.c., where $\Omega$ is the Rabi frequency. Within linear response, the resulting rate of transfer into state $|2\rangle$ is given by $\dot{N}_{2}=-2 \Omega^{2} \operatorname{Im} D\left(\omega_{\text {rf }}\right)$, where $D\left(\omega_{\mathrm{rf}}\right)$ is the Fourier transform of the retarded spin-flip correlation function $D\left(t-t^{\prime}\right)=-i \theta\left(t-t^{\prime}\right)$ $\left\langle\left[\sum_{\mathbf{k}} a_{\mathbf{k} 1}^{\dagger}(t) a_{\mathbf{k} 2}(t), \sum_{\mathbf{k}^{\prime}} a_{\mathbf{k}^{\prime} 2}^{\dagger}\left(t^{\prime}\right) a_{\mathbf{k}^{\prime} 1}\left(t^{\prime}\right)\right]\right\rangle$. Since the BEC of $|1\rangle$ atoms is weakly interacting, i.e., $n_{0} a_{B}^{3} \ll 1$, it can be described up to leading order in $n_{0} a_{B}^{3}$, yielding $D\left(\omega_{\mathrm{rf}}\right)=$ $n_{0} G_{2}\left(\mathbf{k}=\mathbf{0}, \omega_{\text {rf }}\right)$ for a homogenous system, where $G_{2}\left(\mathbf{k}, \omega_{\mathrm{rf}}\right)$ is the Green's function for an atom in spin state $|2\rangle$ with momentum $\hbar \mathbf{k}$ and energy $\hbar \omega_{\mathrm{rf}}$. It follows that an ideal rf measurement directly probes the $\mathbf{k}=\mathbf{0}$ part of the impurity spectral function, defined as $A\left(\omega_{\mathrm{rf}}\right)=-2 \operatorname{Im} G_{2}\left(\mathbf{k}=\mathbf{0}, \omega_{\mathrm{rf}}\right)$. Note that we neglect vertex corrections to $D(\omega)$, which are small for $n_{0} a_{B}^{3} \ll$ 1 as long as the temperature is much smaller than the critical temperature [41].

Subsequent to the rf pulse, the sample is held in the trap for a variable time before being released. After $5 \mathrm{~ms}$ of expansion, a strong magnetic field gradient is applied that separates the $|1\rangle$ and $|2\rangle$ components prior to absorption imaging after a total expansion time of $23 \mathrm{~ms}$. During hold time and expansion, three-body recombination processes involving two $|1\rangle$ and one $|2\rangle$ atom lead to a loss of atoms $[42,43]$. Because of the large atom number imbalance, all $|2\rangle$ atoms are typically lost at strong interactions and the number of $|1\rangle$ atoms is reduced accordingly. By performing a bimodal fit to the spatial distribution of $|1\rangle$ atoms the respective number of atoms in the BEC and thermal cloud is obtained. For strong interactions, we found that the number of lost atoms did not depend on the hold time in the trap and hence the three-body recombination processes during the initial expansion time are sufficient to fully remove $|2\rangle$ atoms. Thus, the hold time was set to 0 for the majority of our measurements.

Because of the three-body recombination process, the number of lost atoms is three times larger than the number of atoms transferred to the polaron state. For each value of the interaction strength, we therefore choose the power of the rf pulse to provide a maximum loss of approximately $30 \%$ corresponding to a polaron fraction of $10 \%$. No significant deviations were observed by moderately varying the polaron fraction, which is discussed in detail in the Supplemental Material [37].

For a given interaction strength $k_{n} a$, we perform spectroscopy on the $|1\rangle \rightarrow|2\rangle$ transition by measuring the resulting $\mathrm{BEC}$ atom number in the $|1\rangle$ state as a function 

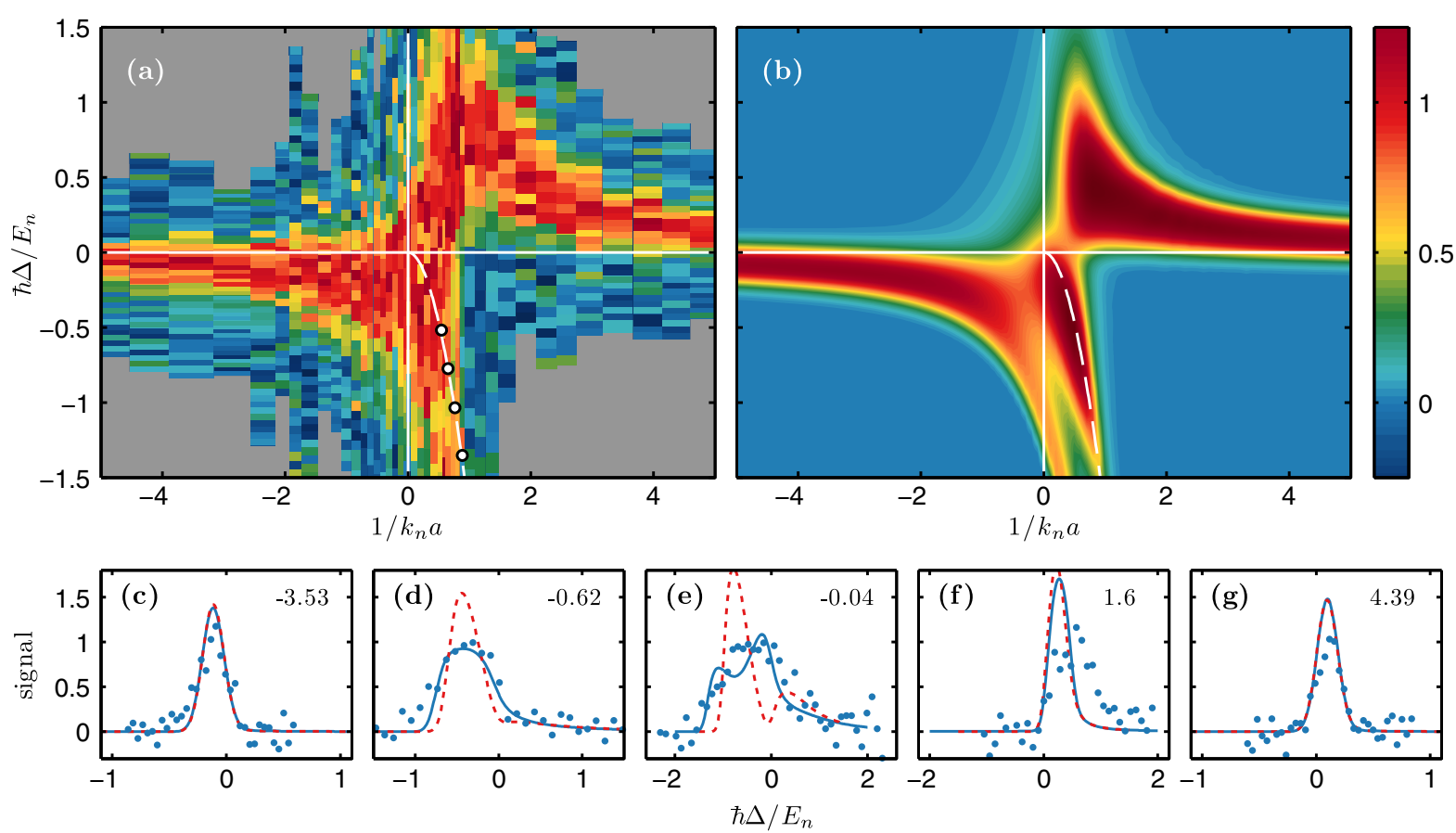

FIG. 2. Spectral response of the impurity in the BEC. The false color plots show the experimentally measured spectroscopic signal (a) and the calculated spectrum (b), for different values of detuning $\Delta$ and the interaction parameter $1 / k_{n} a$. The experimental spectrum is recorded such that its peak amplitude is constant for all values of $1 / k_{n} a$. Accordingly, the theoretical spectrum is normalized such that its frequency integrated weight is the same as the experimental spectrum. In addition, the independently measured molecular binding energy (white dots) and a fit to it (dashed line) are shown [37]. Negative values of the experimental signal are due to shot-to-shot atom number fluctuations. Panels (c)-(g) show the signal as a function of $\Delta$ for various values of $1 / k_{n} a$ (see panel). The solid lines show the calculated signal, which is in excellent agreement with the experiment, except for $1 / k_{n} a=1.6$ where the agreement is qualitative. The dashed lines, obtained excluding three-body correlations, only agree with the experiment for weak interactions.

of detuning $\Delta=\omega_{0}-\omega_{\mathrm{rf}}$, where $\omega_{0}$ is the unperturbed transition frequency between the two states [44]. Figure 2 compares the measured spectroscopic signal, corresponding to the normalized fraction of lost atoms, with that obtained by calculating the spectral function for a zeromomentum impurity using a truncated basis method (TBM) including three-body correlations (see Ref. [45] and the Supplemental Material [37]). The theoretical calculation includes a spatial average over the trapped BEC and a convolution with the Fourier width of the rf pulse. It reproduces the observed signal strikingly well, both for attractive and repulsive interactions. In particular, both experiment and theory show a clear shift in the observed spectral weight due to the interaction between the impurity and the BEC. The calculation of the spectrum involves a restricted Hilbert space of impurity wave functions such that at most two Bogoliubov excitations of the BEC are included. Crucially, this TBM [46] allows us to include three-body correlations in the spectral function nonperturbatively, and thus model the continuum of excited polaron states. Figures $2(\mathrm{c})-2(\mathrm{~g})$ show cuts through the spectrum at fixed $1 / k_{n} a$, demonstrating that the inclusion of three-body correlations is essential for an accurate description of the strongly interacting unitary regime.

In contrast to the Fermi polaron $[6,7,10-13,16]$, there is no sharp transition to a molecular state and the attractive polaron quasiparticle remains the ground state of the system for all interaction strengths. However, the spectral weight of the polaron is increasingly transferred to the continuum of higher-lying states as the strongly interacting unitary regime is approached from the attractive side of the Feshbach resonance. This feature is clearly apparent in both the observed and the calculated spectral response in Fig. 2. For $1 / k_{n} a>0$, the structure of this continuum is determined by the molecular branch, and in the theoretical spectrum we see a clear suppression of spectral weight between the ground-state quasiparticle and the continuum. This is not apparent in the experimental spectrum, potentially due to atom number fluctuations or correlation effects not included in the theory. Significantly, the theory correctly captures the abrupt decrease in the observed signal at negative detuning for $1 / k_{n} a \gtrsim 1$, where the molecule becomes deeply bound compared to $E_{n}$. The detailed comparison of spectroscopic signals in Figs. 2(c)-2(e) further highlights the excellent agreement between theory and experiment for the attractive branch.

To further quantify the results, Fig. 3 compares the average impurity energy obtained from theory and experiment. For the attractive polaron, the experimental data agree well with the results of the TBM. This holds even in the strongly interacting unitarity regime up to and including the abrupt shift of spectral weight to positive detuning at 


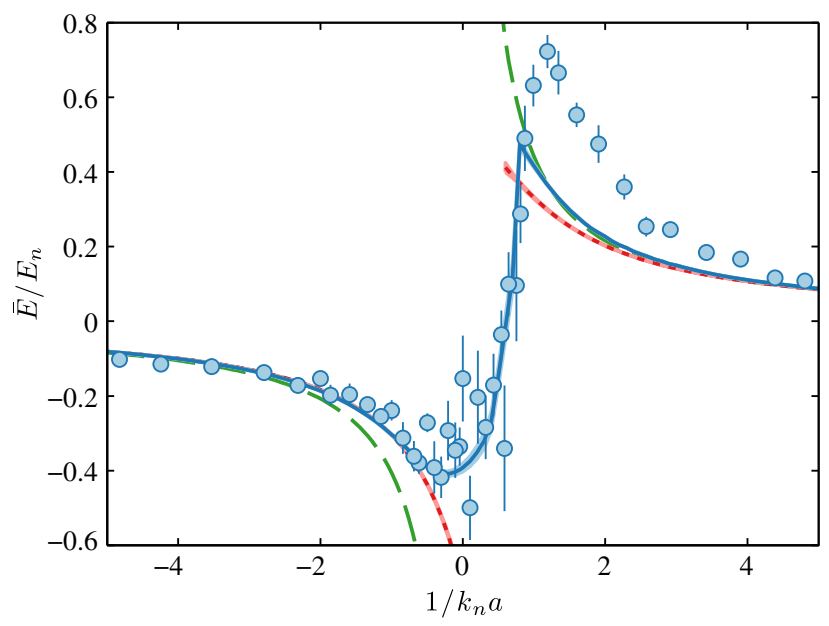

FIG. 3. The average energy $\bar{E}$ of the impurity spectrum is shown as a function of the interaction parameter. The energy was obtained from Gaussian fits to the spectroscopic signal (blue dots) and to the full TBM spectrum (blue line). For comparison, we display the results for a TBM spectrum without three-body correlations (red line) and from perturbation theory (dashed line) [37].

$1 / k_{n} a \simeq 1$. In the case of the repulsive polaron, the agreement is good for weak interaction, whereas there is only qualitative agreement for $1 \lesssim 1 / k_{n} a \lesssim 3$. This suggests that there are important aspects of the experiment that have not been included in the theory, such as effects of temperature, three-body recombination to deeply bound states, and multiple excitations of the BEC. The last effect is likely to play a role for strong interactions near $1 / k_{n} a \simeq 1$, since the repulsive branch in this regime involves a broad continuum of many-body states, which is challenging to model. For comparison, Fig. 3 also includes the result of the TBM without three-body correlations, highlighting the necessity of their inclusion.

Importantly, the perturbative result for the polaron energy [24] accurately reproduces the observed energy shift in Fig. 3 for weak attractive and repulsive interactions. From this we conclude that the experimental data confirm the existence of a well-defined Bose polaron quasiparticle in this regime.

The width of the spectral response also agrees well with theory for interaction parameters $1 / k_{n} a \lesssim 1$ and $1 / k_{n} a \gtrsim 3$, as shown in Fig. 4. For weak interactions, the spectral broadening arises mainly from the Fourier width of the rf pulse and the density inhomogeneity of the trapped BEC. This is illustrated by the fact that the perturbative result assuming a perfect undamped polaron reproduces the observed width in this regime [37]. However, near unitarity where the system is strongly correlated, the spectral weight of the polaron is small, and the many-body continuum of states accounts for the significant broadening of the spectrum. Importantly, this effect is captured by the TBM when three-body correlations are included. For the strongly interacting repulsive branch, there is again only qualitative agreement between theory and experiment for the reasons outlined above.

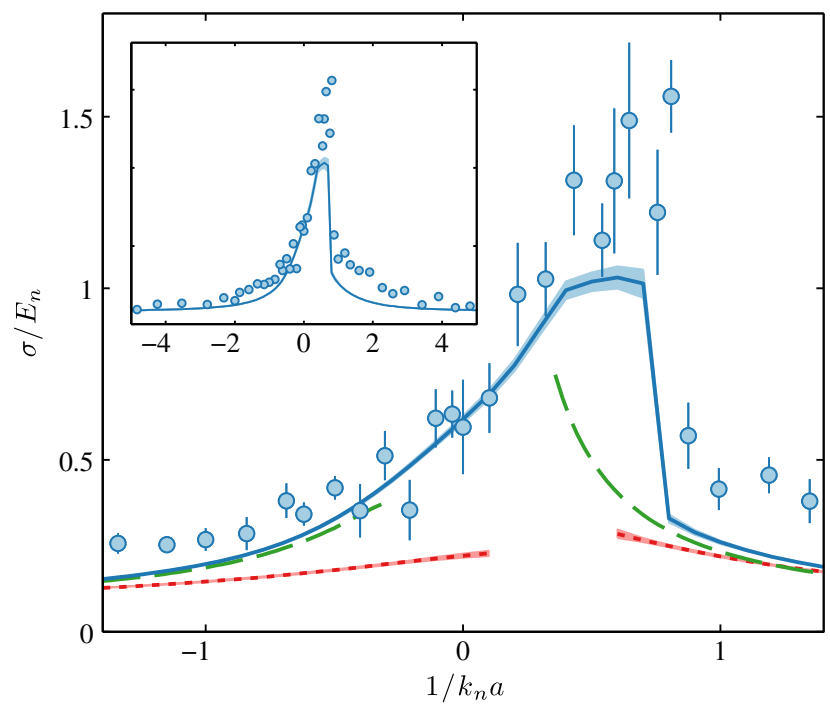

FIG. 4. The width $\sigma$ of the impurity spectrum is shown as a function of the interaction parameter. The widths were obtained from Gaussian fits to the spectroscopic signal (blue dots), the full TBM spectrum (blue line), and the TBM without three-body correlations (red line). The green dashed line was obtained from a spatial average and Fourier width convolution of the result from perturbation theory excluding the many-body continuum [37]. The inset shows the width for the entire experimental data set compared with the full TBM spectrum.

Since strongly interacting Bose systems are expected to suffer from rapid three-body recombination, it is striking how well the experimental observations are described by theories that neglect such losses. The observed width of the spectrum is explained by the trap inhomogeneity, Fourier broadening, and many-body continuum. Thus, while our method of detection relies on three-body losses, our results demonstrate that these processes occur on time scales longer than those associated with polaron physics. Therefore, the polaron is not significantly affected by three-body decay, indicating that it is long lived. We note that the impurity decay rate, which is proportional to $n_{0}^{2} a^{4}$ when $n_{0}|a|^{3} \ll 1$, is ultimately limited by the average interparticle spacing in the unitary regime. In this case the energy shift and decay rate both scale as $n_{0}^{2 / 3}$. Our results thus imply that the ratio of the decay rate to the energy shift at unitarity remains small, a finding that is consistent with the recent experiment on the unitary Bose gas [47].

In conclusion, using rf spectroscopy on a BEC of ${ }^{39} \mathrm{~K}$ atoms, we observed the existence of a long-lived Bose polaron. The spectral response is characterized by a welldefined quasiparticle peak both for weak attractive and repulsive interactions, whereas it becomes strongly broadened due to many-body effects for stronger interaction. We found excellent agreement with theoretical results including three-body correlations, even for strong coupling. Our observation of a well-defined Bose polaron opens up the exciting opportunity to study quantum impurities in a bosonic environment systematically and in regimes never 
realized before. For instance, an intriguing question is how the polaron changes when the BEC melts. The effects of such a phase transition of the environment on an impurity particle have never been investigated before. Also, it will be interesting to examine the dynamics of the polaron and effects such as momentum relaxation, as well as induced interactions between polarons and the formation of multipolaron states. There is also the prospect of observing stable Efimov trimers in a many-body environment for the first time $[25,48,49]$. We do not expect to observe this in our present experiment, since the size of the smallest Efimov trimer is estimated to be 100 times larger than the interparticle spacing [25]. However, the Efimovian regime can be accessed by lowering the density or by using light impurities.

N. B. J., L. W., K. T. S., and J. J. A. acknowledge support from the Lundbeck Foundation and the Danish Council for Independent Research. R. S. C. and G. M. B. acknowledge support from the Villum Foundation via Grant No. VKR023163.

Note added.-Note that parallel experiments were performed in a Bose-Fermi mixture, and report similar results [33].

[1] L. D. Landau and S. I. Pekar, J. Exp. Theor. Phys. 18, 419 (1948).

[2] G. Mahan, Many-Particle Physics (Kluwer Academic/ Plenum Publishers, New York, 2000).

[3] M. E. Gershenson, V. Podzorov, and A. F. Morpurgo, Rev. Mod. Phys. 78, 973 (2006).

[4] G. Baym and C. Pethick, Landau Fermi-Liquid Theory: Concepts and Applications (Wiley-VCH, New York, 1991).

[5] E. Dagotto, Rev. Mod. Phys. 66, 763 (1994).

[6] A. Schirotzek, C.-H. Wu, A. Sommer, and M. W. Zwierlein, Phys. Rev. Lett. 102, 230402 (2009).

[7] C. Kohstall, M. Zaccanti, M. Jag, A. Trenkwalder, P. Massignan, G. M. Bruun, F. Schreck, and R. Grimm, Nature (London) 485, 615 (2012).

[8] M. Koschorreck, D. Pertot, E. Vogt, B. Fröhlich, M. Feld, and M. Köhl, Nature (London) 485, 619 (2012).

[9] F. Chevy, Phys. Rev. A 74, 063628 (2006).

[10] N. Prokof'ev and B. Svistunov, Phys. Rev. B 77, 020408 (2008).

[11] C. Mora and F. Chevy, Phys. Rev. A 80, 033607 (2009).

[12] M. Punk, P. T. Dumitrescu, and W. Zwerger, Phys. Rev. A 80, 053605 (2009).

[13] R. Combescot, S. Giraud, and X. Leyronas, Europhys. Lett. 88, 60007 (2009).

[14] X. Cui and H. Zhai, Phys. Rev. A 81, 041602 (2010).

[15] C. J. M. Mathy, M. M. Parish, and D. A. Huse, Phys. Rev. Lett. 106, 166404 (2011).

[16] P. Massignan, M. Zaccanti, and G. M. Bruun, Rep. Prog. Phys. 77, 034401 (2014).

[17] F. M. Cucchietti and E. Timmermans, Phys. Rev. Lett. 96, 210401 (2006).
[18] B.-B. Huang and S.-L. Wan, Chin. Phys. Lett. 26, 080302 (2009).

[19] J. Tempere, W. Casteels, M. K. Oberthaler, S. Knoop, E. Timmermans, and J. T. Devreese, Phys. Rev. B 80, 184504 (2009).

[20] S. P. Rath and R. Schmidt, Phys. Rev. A 88, 053632 (2013).

[21] W. Li and S. Das Sarma, Phys. Rev. A 90, 013618 (2014).

[22] F. Grusdt, Y. E. Shchadilova, A. N. Rubtsov, and E. Demler, Sci. Rep. 5, 12124 (2015).

[23] L. A. Pena Ardila and S. Giorgini, Phys. Rev. A 92, 033612 (2015).

[24] R. S. Christensen, J. Levinsen, and G. M. Bruun, Phys. Rev. Lett. 115, 160401 (2015).

[25] J. Levinsen, M. M. Parish, and G. M. Bruun, Phys. Rev. Lett. 115, 125302 (2015).

[26] N. Spethmann, F. Kindermann, S. John, C. Weber, D. Meschede, and A. Widera, Phys. Rev. Lett. 109, 235301 (2012).

[27] C. Zipkes, S. Palzer, C. Sias, and M. Kohl, Nature (London) 464, 388 (2010).

[28] S. Schmid, A. Härter, and J. H. Denschlag, Phys. Rev. Lett. 105, 133202 (2010).

[29] J. B. Balewski, A. T. Krupp, A. Gaj, D. Peter, H. P. Buchler, R. Low, S. Hofferberth, and T. Pfau, Nature (London) 502, 664 (2013).

[30] R. Scelle, T. Rentrop, A. Trautmann, T. Schuster, and M. K. Oberthaler, Phys. Rev. Lett. 111, 070401 (2013).

[31] G. E. Marti, A. MacRae, R. Olf, S. Lourette, F. Fang, and D. M. Stamper-Kurn, Phys. Rev. Lett. 113, 155302 (2014).

[32] S. Ospelkaus, C. Ospelkaus, O. Wille, M. Succo, P. Ernst, K. Sengstock, and K. Bongs, Phys. Rev. Lett. 96, 180403 (2006).

[33] M.-G. Hu, M. J. Van de Graaff, D. Kedar, J. P. Corson, E. A. Cornell, and D. S. Jin, preceding Letter, Phys. Rev. Lett. 117, 055301, (2016)

[34] V. Efimov, Phys. Lett. 33B, 563 (1970).

[35] E. Braaten and H.-W. Hammer, Phys. Rep. 428, 259 (2006).

[36] T. Kraemer, M. Mark, P. Waldburger, J. G. Danzl, C. Chin, B. Engeser, A. D. Lange, K. Pilch, A. Jaakkola, H.-C. Nägerl, and R. Grimm, Nature (London) 440, 315 (2006).

[37] See Supplemental Material http://link.aps.org/supplemental/ 10.1103/PhysRevLett.117.055302 for additional details on the experiments and the theoretical modeling.

[38] L. Wacker, N. B. Jørgensen, D. Birkmose, R. Horchani, W. Ertmer, C. Klempt, N. Winter, J. Sherson, and J. J. Arlt, Phys. Rev. A 92, 053602 (2015).

[39] M. Lysebo and L. Veseth, Phys. Rev. A 81, 032702 (2010).

[40] M. W. Zwierlein, Z. Hadzibabic, S. Gupta, and W. Ketterle, Phys. Rev. Lett. 91, 250404 (2003).

[41] C. J. Pethick and H. T. C. Stoof, Phys. Rev. A 64, 013618 (2001).

[42] B. D. Esry, C. H. Greene, and J. P. Burke, Phys. Rev. Lett. 83, 1751 (1999).

[43] E. Nielsen and J. H. Macek, Phys. Rev. Lett. 83, 1566 (1999).

[44] At weak and moderate interactions, the signal obtained from the thermal atoms confirms the position of the bare transition $\omega_{0}$ that is obtained from independent measurements. 
[45] M. M. Parish and J. Levinsen (to be published).

[46] M. Cetina, M. Jag, R. S. Lous, I. Fritsche, J. T. M. Walraven, R. Grimm, J. Levinsen, M. M. Parish, R. Schmidt, M. Knap, and E. Demler, arXiv:1406.6506.
[47] P. Makotyn, C. E. Klauss, D. L. Goldberger, E. A. Cornell, and D. S. Jin, Nat. Phys. 10, 116 (2014).

[48] Y. Nishida, Phys. Rev. Lett. 114, 115302 (2015).

[49] W. Yi and X. Cui, Phys. Rev. A 92, 013620 (2015). 